Yamaguchi, E. and T. Nishioka, Cold fusion induced by controlled out-diffusion of deuterons in palladium. Jpn. J. Appl. Phys. Part 2, 1990. 29(4): p. L666.

\title{
Cold Nuclear Fusion Induced by Controlled Out-Diffusion of Deuterons in Palladium
}

\author{
Eiichi YAMAGUCHI and Takashi NISHIOKA \\ NTT Basic Research Laboratories, Musashino-shi, Tokyo 180 \\ (Received January 24, 1990; accepted for publication March 7, 1990)
}

\begin{abstract}
A gigantic neutron burst of $(1-2) \times 10^{6} \mathrm{n} / \mathrm{s}$ has been detected from deuterated Pd plates with heterostructures set in a vacuum chamber. An explosive release of $\mathrm{D}_{2}$ gas, biaxial bending of all the samples, and excess heat evolution were also observed at the same time. It has been concluded that these phenomena are caused by the cooperative production of D accumulation layers at Pd surfaces due to controlled out-diffusion of D-atoms.
\end{abstract}

KEYWORDS: cold nuclear fusion, deuteron, deuterium gas, palladium, heterostructure, out-diffusion, neutron emission, biaxial strain, heat evolution, cooperative phenomenon

Since Jones et al. ${ }^{1\}}$ and Fleischmann and Pons ${ }^{2)}$ reported that nuclear fusion occurred at room temperature in Pd or Ti cathodes during the electrolysis of $\mathrm{D}_{2} \mathrm{O}$, much effort has been made to reinvestigate the possibility of the electrolytically induced cold fusion in condensed matter. ${ }^{3-12)}$ Spontaneous neutron emission due to the fusion of D-atoms has also been observed during the process of soaking Ti powder into $\mathrm{D}_{2}$ gas up to $40 \mathrm{~atm}$, followed by cooling with liquid nitrogen and heating. ${ }^{13)}$ More recently, it has been reported that $\mathrm{D}^{+}$ions accelerated to more than $1 \mathrm{keV}$ caused fusion at a high rate in deuterated $\mathrm{Ti}^{14)}$ or $\mathrm{Pd}^{15)}$ Although the "high-energy" nuclear fusion in solids can be well understood within the current theory because the fusion rate at $1 \mathrm{keV}$ has been calculated to be more than $10^{13}$ times larger than that at $300 \mathrm{eV}$ or less, ${ }^{*}$ the observed neutron emission without application of high voltages ${ }^{15)}$ has given evidence of cold nuclear fusion. In this letter, we report a new technique for inducing "low-energy" nuclear fusion at room temperature in solids. By making use of this technique, we have observed a gigantic neutron burst and, at the same time, an explosive release of $\mathrm{D}_{2}$ gas from deuterated Pd (Pd:D) plates as well as a biaxial bending of the samples. We have also detected excess heat evolution.

The key process in the present study is the formation of $\mathrm{D}$ accumulation layers at solid surfaces by controlling the D-atom out-diffusive transport with hetrostructures. Namely, one of the surfaces of a Pd:D ( $\alpha$-phase) substrate is covered with a thick $\mathrm{Au}$-film in order to prevent the leakage of D-atoms from this side. In the present experiment, the substrates were obtained by immersing annealed Pd plates $(99.9 \%$; thickness $=1.0 \mathrm{~mm})$ into $\mathrm{D}_{2}$ gas $(99.9 \%$; $0.5 \mathrm{~atm})$ for 24 hours. The other surface is covered with a thin film having a diffusion constant of D less than Pd. This layer appropriately controls the out-diffusion of D-atoms passing through this interface. In the present work, we used a film with a thickness of less than $100 \AA$ A containing mainly $\mathrm{Mn}$ and $\mathrm{O}$, which we call $\mathrm{Mn}-\mathrm{O}$ film in this letter. Thus, the $\mathrm{D}$ accumulation layer can be formed at the interface during the out-diffusion processes of D-atoms by decreasing the pressure of ambient $\mathrm{D}_{2}$ gas. It will be shown later that the formation of the $\beta$-phase layer can provide cooperative feedback to cause further accumulation of D-atoms.

As soon as the sample preparation was completed, we set three of these samples in another stainless-steel chamber, as schematically shown in Fig. 1, and evacuated it. Neutrons were counted by using a BF 3 detector (Aloka Co., Ltd: TPS-451S) set at $38 \mathrm{~cm}$ from the samples. The leak detector (Varian Ltd: 925-40), monitoring gases of mass numbers less than 6, was also set in the vacuum chamber. About three hours after pumping, we observed the virtually simultaneous occurrence of the following events: (1) neutron emission of 0.1-0.2 mSv/h for 2-3 seconds, (2) explosive release of gas from the samples, and (3) biaxial bending of all the samples due to uniform expansion of the surfaces with a thin Mn-O film (see Fig. 2). When the samples were taken out from the chamber, we found an increase in the temperature of about $50^{\circ} \mathrm{C}$ on the stainless-steel sample holder as well as on all three samples. We also observed that the color of Au deposited on one of the surfaces was lost, which indicated alloying of $\mathrm{Au}$ and Pd. Thus, it is supposed that the temperature on the samples were temporarily increased to above the Au-Pd alloying point $\left(1064^{\circ} \mathrm{C}\right) .^{16)}$

\footnotetext{
* J. Rafelski, M. Gajda, D. Harley and S. E. Jones: private communication.
} 

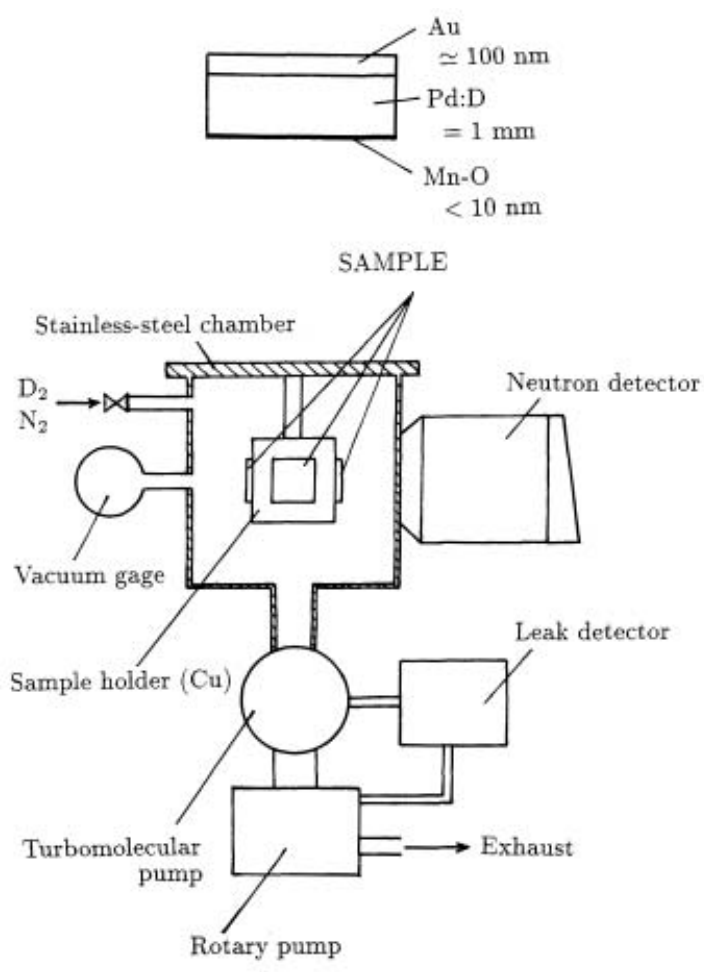

Fig. 1. Schematic diagram of the measurement apparatus.

In order to check the reproducibility on the same samples, we again immersed these samples into $\mathrm{D}_{2}$ gas in the same chamber, and then evacuated it. About 150 seconds after the start of pumping, we observed a second gigantic neutron burst of 0.06-0.09 $\mathrm{mSv} / \mathrm{h}$ for 1-2 seconds followed by an explosive release of gas. After the pressure in the chamber was increased up to $1 \mathrm{~atm}$ with $\mathrm{N}_{2}$, we again evacuated the chamber. Almost the same neutron emission and gas release were again observed about 150 seconds after the start of pumping. The second and third neutron bursts were recorded by the analog output of the neutron detector and are shown in Fig. 3.

We performed approximately 20 experiments with the same procedure as denoted above. Neither of the above-mentioned events was observed in any of those experiments. We also performed a few experiments replacing $\mathrm{D}_{2}$-gas by $\mathrm{H}_{2}$-gas and observed neither explosive gas release nor neutron emission. However, this does not mean that a mechanism responsible for the observed phenomena other than the fusion reaction itself does not exist in Pd:H systems, because the production of accumulation layers followed by biaxial bending of the samples is expected to be almost independent of the mass number of the hydrogen-isotope.

Here, it must be noted that all high-voltage $(>200 \mathrm{~V})$ power sources in the laboratory, except for the ion vacuum gauge, were off when the first, second and third neutron emissions described above were detected. We used batteries as the power source of the neutron detectors throughout the present experiment, and continuously confirmed the absence of a noise on the analog output of the detectors, for a few months since the beginning of the experiment. It must further be noted that, in order to calibrate the $\mathrm{BF}_{3}$ neutron detector, we performed $\mathrm{D}^{+}$-ion implantation into Pd:D at 50 to $200 \mathrm{keV}$ just after the first and second neutron emissions. It was found that the measured neutron flux coincides with that measured by another neutron detector (Nuclear Enterprises Ltd.: NM2B) at various acceleration energies and dose rates.

Provided that event (1) results from the usual D-D fusion reaction, the energy of emitted neutrons is $2.5 \mathrm{MeV}$ and/or 14.1 $\mathrm{MeV}$. Then, the neutron dose of $0.1 \mathrm{mSv} / \mathrm{h}$ is equivalent to the neutron flux of $72 \mathrm{n} /\left(\mathrm{cm}^{2} \cdot \mathrm{s}\right){ }^{17)}$ Thus, the amplitude of the first neutron emission is roughly evaluated to be $(1-2) \times 10^{6} \mathrm{n} / \mathrm{s}$ by assuming the neutron emission from the samples to be almost independent of the angle. The amplitude of the second and third emissions is equivalent to $(0.6-0.9) \times 10^{6} \mathrm{n} / \mathrm{s}$. The neutron flux of the first emission is (2.5-5) $\times 10^{6}$ times larger than that reported by Jones et al. ${ }^{1\}}$ and as much as 25-50 times larger than that reported by Fleischmann and Pons. ${ }^{2}$ Here, it is noted that the background level for neutron detection was $\sim(1-3) \times$ $10^{-9} \mathrm{~Sv} / \mathrm{h}\left(\sim 10^{-3} \mathrm{n} /\left(\mathrm{cm}^{2} \cdot \mathrm{s}\right)\right)$ throughout the present investigation. 


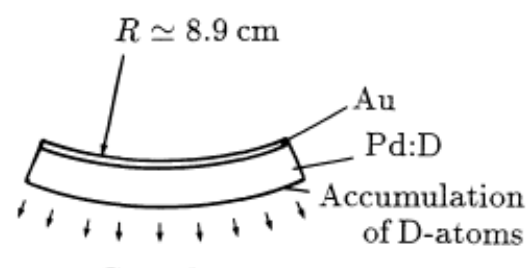

Gas release

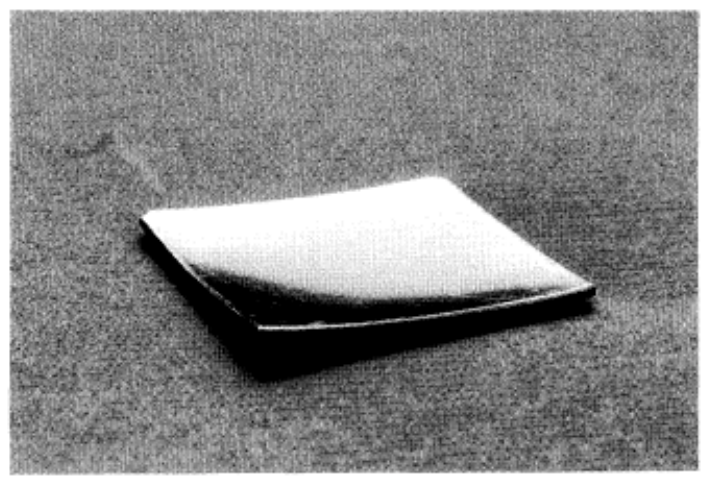

Fig. 2. A $3.0 \mathrm{~cm} \times 3.0 \mathrm{~cm}$ palladium sample (thickness $=1.0 \mathrm{~mm}$ ) after the third neutron burst and explosive release of $\mathrm{D}_{2}$ gas. Top side is Au-coated surface at which alloying occurred after the first neutron emission.

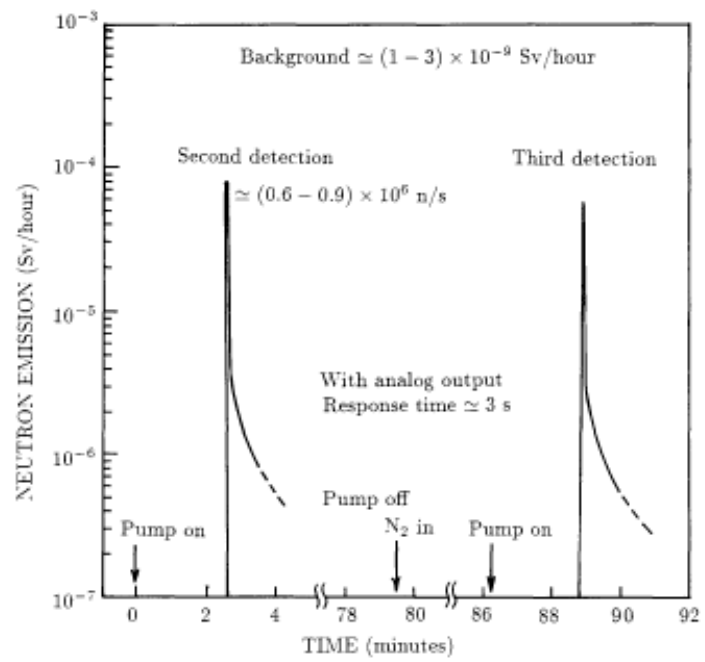

Fig. 3. The second and third neutron emissions as a function of time. The tailing behaviour after the neutron is detected is of no significance; it is only a characteristic of the analog output of the device.

Furthermore, it can be considered that the gas released in event (2) is $\mathrm{D}_{2}$ which was dissolved in the Pd samples, because atoms or molecules with a mass number of 4 were detected in great quantity in the residual gas. Here, it is noted that atoms or molecules with the mass number of 3 were slightly detected. Since the leakage of D-atoms from Au-coated surfaces can be ignored, event (2) is thought to be caused mainly by explosive out-diffusion of D-atoms through the interface between Pd and a thin $\mathrm{Mn}-\mathrm{O}$ film. Besides, the reason for the sudden excess heat production is still not clear. It may be strongly correlated to the decomposition of $\operatorname{PdD}_{\mathrm{x}}(x>0.6)$ and subsequent release of $\mathrm{D}_{2}$ gas, as argued by Pauling, ${ }^{18)}$ or to the lattice rearrangement due to the plastic deformation, as described by event (3).

Now let us consider the origin of the enhancement of the nuclear fusion rate observed in the present experiment. Kondo, ${ }^{19)}$ Sun and Tomanek ${ }^{20)}$ and Wang et al. ${ }^{21\}}$ have theoretically shown that the separation between hydrogen atoms is much larger than the value required for a significant fusion rate in the equilibrium. Therefore it is believed that the present 
observation should result from some nonequilibrium processes, for instance, a rapid change in the configuration of host Pdatoms or defects so as to bring D-atoms much closer to each other than in the equilibrium sites.

One possibility for this is the "fracture" mechanism; ${ }^{22-25)}$ that is, an electrostatic field induced at the fracture or crack may accelerate the $\mathrm{D}^{+}$-ions and cause nuclear fusion. Another possible mechanism is the host-lattice rearrangement due to sudden plastic deformation followed by dynamical "musical-chairs" motions of D-atoms as follows:

During the diffusion process, D-atoms are accumulated at the interface and form a thin layer of $\beta$-phase $\mathrm{PdD}_{\mathrm{x}}(0.6<x<$ 1.0), or an oversaturation phase with $x$ larger than 1. This formation increases the lattice constant at this layer, and then gives a biaxial strain to the samples. The strain, in turn, enhances the out-diffusion of D-atoms to the same side of the surfaces (Gorsky effect). Therefore, these effects can give a cooperative cycle of positive feedback to increase the D concentration at that side of Pd surfaces. ${ }^{*}$ The resulting catastrophic increase in the strain due to the accumulation of D-atoms finally produces a plastic deformation in the sample plates, as in event (3), and causes each host Pd-atom to rearrange rapidly. As a consequence, D-atoms are forced to move dynamically to attain new potential minima. In fact, the X-ray analysis for the samples after the third neutron emission has revealed that there were considerable degradations of Pd crystals without the $\beta$ phase at the expanded surfaces, while the Au-coated surfaces had no degradation and contained a slight amount of $\beta$-phase.

Here, we will conjecture that the rapid rearrangement of Pd-atoms can greatly increase the potential energy of D-atoms, by showing a simple example. Figure 4 indicates the calculated contour map of the empirical potential ${ }^{26)}$ for the H-atom in Pd. As shown in Fig. 4(a), both the octahedral (O)-sites and the tetrahedral (T)-sites, whose numbers are, respectively, 1 and 2 for one Pd-atom, give local minima without strain. As the biaxial strain parallel to the (110) plane is increased, the O-sites tend to become saddle points and no longer offer stable sites to hydrogen atoms, as shown in Fig. 4(b). In this case, the displaced Tsites become new local minima. Within such elastic deformation, it cannot be expected that $\mathrm{D}$-atoms, which could have moved from the $\mathrm{O}$-sites to the displaced $\mathrm{T}$-sites, obtain energy high enough to give a high fusion reaction. However, at the very moment of plastic deformation, the Pd-atom rearrangement due to the production of defects can increase the potential magnitude at the displaced T-sites to the order of $10^{2-3} \mathrm{eV}$, as schematically shown in Fig. 4(c) for the simplest defect (a single vacancy). It can be easily shown that this also holds for more complex defects such as edge or screw dislocations. Here, it must be noted that the number of potential minima can be decreased for any case. Therefore, at this time, the probability that D-atoms having the kinetic energy of the order of $10^{2-3} \mathrm{eV}$ collide with each other will be increased significantly. More detailed calculations will be necessary to check the validity of the proposed model.

\footnotetext{
${ }^{*}$ The difficulty in reproducing the present experiment may therefore be due to the fact that the cooperative production of D accumulation layers at Pd surfaces critically depends on the characteristics of the surface barriers.
} 
(a)
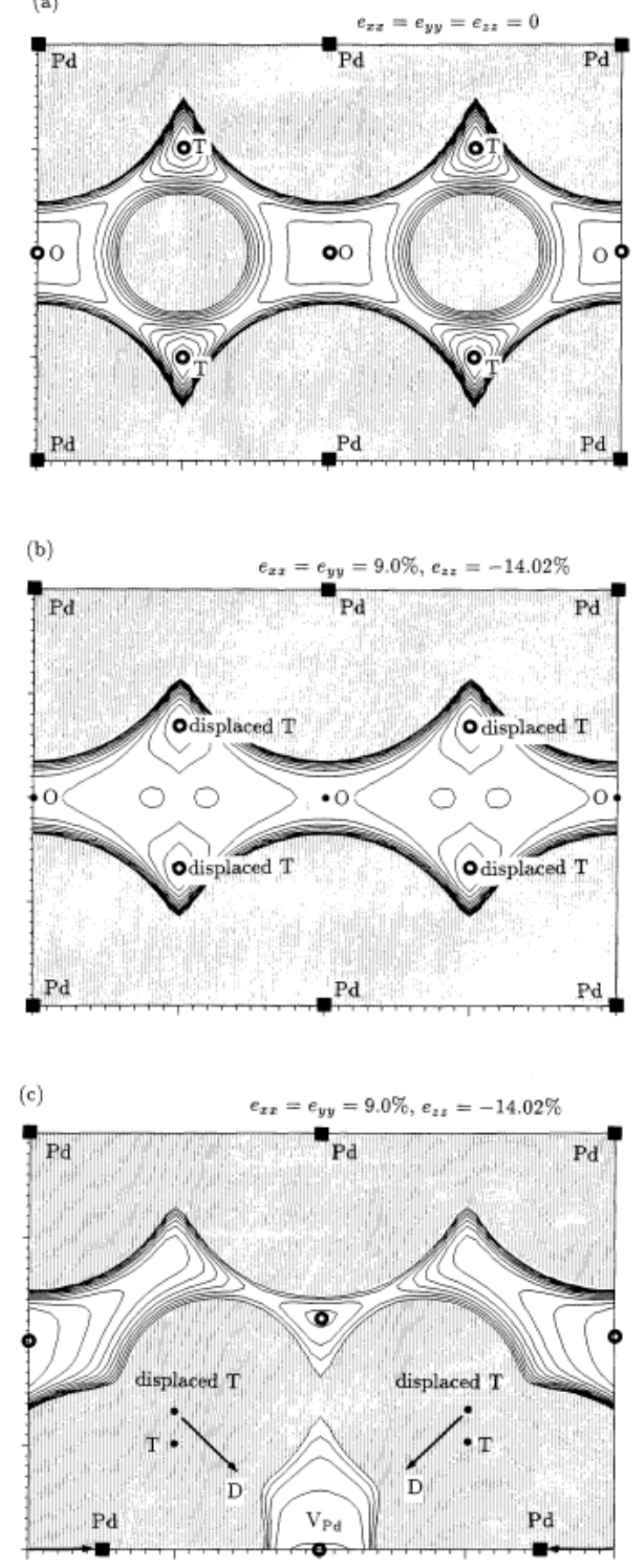

Fig. 4. Potential contour for the $\mathrm{H}$-atom in the $\mathrm{Pd}(110)$ plane (a) without the strain, (b) with the biaxial strain $\left(e_{x x}=e_{y y}=9 \%\right)$ and $(c)$ with a vacancy $\left(\mathrm{V}_{\mathrm{Pd}}\right)$ and nearest-neighbor rearrangement (bond-shrink by 25\%) as well as the biaxial strain, where the $\langle 110\rangle$ direction is set to the z-axis and the contour step is $50 \mathrm{meV}$ (200 meV for Fig. 4(c)). Shaded areas have energies larger than $500 \mathrm{meV}$ ( $2 \mathrm{eV}$ for Fig. 4(c)) from the potential bottom, and the open circles represent the local minima of the potential. 


\section{Acknowledgements}

We are grateful to Dr. Tatsuya Kimura for his very useful advice. We also thank Drs. Kiyomasa Sugii, Kenju Otsuka, Yoshiji Horikoshi and Takahisa Ohno for helpful discussions.

\section{References}

1) S. E. Jones, E. P. Palmer, J. B. Czirr, D. L. Decker, G. L. Jensen, J. M. Thome, S. F. Taylor and J. Rafelski: Nature 338 (1989) 737.

2) M. Fleischmann and S. Pons: J. Electroanal. Chem. 261 (1989) 301.

3) A. Baurichter, W. Eyrich, M. Frank, H. Gohr, W. Krische, H. Ortner, B. Roseler, C. A. Schiller, G. Weeske and W. Witthun: Z. Phys. B76(1989) 1.

4) G. Kreysa, G. Marx and W. Plieth: J. Electroanal. Chem. 266 (1989) 437.

5) S. Feng: Solid State Commun. 72 (1989) 205.

6) D. Alber, O. Boebel, C. Schwartz, H. Duwe, D. Hilscher, H. Homeyer, U. Jahnke and B. Spellmeyer: Z. Phys. A333 (1989) 319.

7) S. Bragus, M. Bogovac, D. Hodko, M. Krcmar, D. Miljanic, P. Tomas, M. Vajic and M. Vukovic: Z. Phys. A333 (1989) 321.

8) M. Gai, S. L. Rugari, R. H. France, B. J. Lund, Z. Zhao, A. J. Davenport, H. S. Isaac and K. G. Lynn: Nature 340 (1989) 29.

9) N. S. Lewis, C. A. Barnes, M. J. Heben, A. Kumar, S. R. Lunt, G. E. Me- Manis, G. M. McManis, G. M. Miskelly, R. M. Penner, M. J. Sailor, P. G. Santangelo, G. A. Shreve, B. J. Tufts, M. G. Youngquist, R. W. Kavanagh, S. E. Kellogg, R. B. Vogelaar, T. R. Wang, R. Kondrat and R. New: Nature 340 (1989) 525.

10) P. B. Price, S. W. Barwick, W. T. Williams and J. D. Porter: Phys. Rev. Lett. 63 (1989) 1926.

11) D. E. Williams, D. J. S. Findlay, D. H. Craston, M. R. Sene, M. Bailey, S. Croft, B. W. Hooton, C. P. Jones, A. R. J. Kucernak, J. A. Mason and R. I. Taylor: Nature 342 (1989) 23.

12) R. Taniguchi, T. Yamamoto and S. Irie: Jpn. J. Appl. Phys. 28 (1989) L2021.

13) A. De Ninno, A. Frattolillo, G. Lollobattista, L. Martinis, M. Martone, L. Mori, S. Podda and F. Scaramuzzi: Nuovo Cimento A 101 (1989) 841.

14) R. J. Beuhler, G. Friedlander and L. Friedman: Phys. Rev. Lett. 63 (1989) 1292.

15) N. Wada and K. Nishizawa: Jpn. J. Appl. Phys. 28 (1989) L2017. M. Hansen and K. Anderko: Constitution of Binary Alloys (McGraw-Hill, New York, 1985) $2^{\text {nd }}$ ed., p. 224.

16) A. Martin and S. A. Harbison: An Introduction to Radiation Protection (Chapman and Hall Ltd., London, 1972).

17) L. Pauling: Nature 339 (1989) 105.

18) J. Kondo: J. Phys. Soc. Jpn. 58 (1989) 1869.

19) Z. Sun and D. Tomanek: Phys. Rev. Lett. 63 (1989) 59.

20) X. W. Wang, S. G. Louie and M. L. Cohen: Phys. Rev. B40 (1989) 5822.

21) T. Takeda and T. Takizuka: J. Phys. Soc. Jpn. 58 (1989) 3073.

22) B. V. Derjaguin, A. G. Lipson, V. A. Kluev, D. M. Sakov and Y. P. Toporov: Nature 341 (1989) 492.

23) J. S. Cohen and J. D. Davies: Nature 342 (1989) 487.

24) J. T. Dickinson, L. C. Jensen, S. C. Langford, R. R. Ryan and E. Garcia: J. Mater. Res. 5 (1990) 109.

25) H. Sugimoto and Y. Fukai: J. Phys. Soc. Jpn. 51 (1982) 2554. 